\title{
Rarefied sets at infinity associated with the Schrödinger operator
}

\section{Gaixian Xue*}

\section{"Correspondence:} jingben84@163.com

School of Mathematics and Information Science, Henan University of Economics and Law, Zhengzhou, 450046, China

\begin{abstract}
This paper gives some criteria for a-rarefied sets at infinity associated with the Schrödinger operator in a cone. Our proofs are based on estimating Green a-potential with a positive measure by connecting with a kind of density of the modified measure. Meanwhile, the geometrical property of this a-rarefied sets at infinity is also considered. By giving an example, we show that the reverse of this property is not true.
\end{abstract}

Keywords: rarefied set; Schrödinger operator; Green a-potential

\section{Introduction and results}

Let $\mathbf{R}$ and $\mathbf{R}_{+}$be the set of all real numbers and the set of all positive real numbers, respectively. We denote by $\mathbf{R}^{n}(n \geq 2)$ the $n$-dimensional Euclidean space. A point in $\mathbf{R}^{n}$ is denoted by $P=\left(X, x_{n}\right), X=\left(x_{1}, x_{2}, \ldots, x_{n-1}\right)$. The Euclidean distance between two points $P$ and $Q$ in $\mathbf{R}^{n}$ is denoted by $|P-Q|$. Also $|P-O|$ with the origin $O$ of $\mathbf{R}^{n}$ is simply denoted by $|P|$. The boundary and the closure of a set $S$ in $\mathbf{R}^{n}$ are denoted by $\partial S$ and $\bar{S}$, respectively.

We introduce a system of spherical coordinates $(r, \Theta), \Theta=\left(\theta_{1}, \theta_{2}, \ldots, \theta_{n-1}\right)$, in $\mathbf{R}^{n}$ which are related to Cartesian coordinates $\left(x_{1}, x_{2}, \ldots, x_{n-1}, x_{n}\right)$ by $x_{n}=r \cos \theta_{1}$.

Let $D$ be an arbitrary domain in $\mathbf{R}^{n}$ and let $\mathscr{A}_{a}$ denote the class of non-negative radial potentials $a(P)$, i.e., $0 \leq a(P)=a(r), P=(r, \Theta) \in D$, such that $a \in L_{\text {loc }}^{b}(D)$ with some $b>n / 2$ if $n \geq 4$ and with $b=2$ if $n=2$ or $n=3$.

If $a \in \mathscr{A}_{a}$, then the Schrödinger operator

$$
\operatorname{Sch}_{a}=-\Delta+a(P) I=0
$$

where $\Delta$ is the Laplace operator and $I$ is the identical operator, can be extended in the usual way from the space $C_{0}^{\infty}(D)$ to an essentially self-adjoint operator on $L^{2}(D)$ (see [1, Ch. 11]). We will denote it by $S_{c h}$ as well. This last one has a Green $a$-function $G_{D}^{a}(P, Q)$. Here $G_{D}^{a}(P, Q)$ is positive on $D$ and its inner normal derivative $\partial G_{D}^{a}(P, Q) / \partial n_{Q} \geq 0$, where $\partial / \partial n_{Q}$ denotes the differentiation at $Q$ along the inward normal into $D$.

We call a function $u \neq \equiv-\infty$ that is upper semi-continuous in $D$ a subfunction with respect to the Schrödinger operator $S c h_{a}$ if its values belong to the interval $[-\infty, \infty)$ and at each point $P \in D$ with $0<r<r(P)$ the generalized mean-value inequality (see [2])

$$
u(P) \leq \int_{S(P, r)} u(Q) \frac{\partial G_{B(P, r)}^{a}(P, Q)}{\partial n_{Q}} d \sigma(Q)
$$

( 2014 Xue; licensee Springer. This is an Open Access article distributed under the terms of the Creative Commons Attribution License (http://creativecommons.org/licenses/by/2.0), which permits unrestricted use, distribution, and reproduction in any medium, provided the original work is properly cited. 
is satisfied, where $G_{B(P, r)}^{a}(P, Q)$ is the Green $a$-function of $S_{c h}$ in $B(P, r)$ and $d \sigma(Q)$ is a surface measure on the sphere $S(P, r)=\partial B(P, r)$.

If $-u$ is a subfunction, then we call $u$ a superfunction. If a function $u$ is both subfunction and superfunction, it is, clearly, continuous and is called an $a$-harmonic function (with respect to the Schrödinger operator $S c h_{a}$ ).

The unit sphere and the upper half unit sphere in $\mathbf{R}^{n}$ are denoted by $\mathbf{S}^{n-1}$ and $\mathbf{S}_{+}^{n-1}$, respectively. For simplicity, a point $(1, \Theta)$ on $\mathbf{S}^{n-1}$ and the set $\{\Theta ;(1, \Theta) \in \Omega\}$ for a set $\Omega, \Omega \subset \mathbf{S}^{n-1}$, are often identified with $\Theta$ and $\Omega$, respectively. For two sets $\Xi \subset \mathbf{R}_{+}$and $\Omega \subset \mathbf{S}^{n-1}$, the set $\left\{(r, \Theta) \in \mathbf{R}^{n} ; r \in \Xi,(1, \Theta) \in \Omega\right\}$ in $\mathbf{R}^{n}$ is simply denoted by $\Xi \times \Omega$. By $C_{n}(\Omega)$ we denote the set $\mathbf{R}_{+} \times \Omega$ in $\mathbf{R}^{n}$ with the domain $\Omega$ on $\mathbf{S}^{n-1}$. We call it a cone. We denote the set $I \times \Omega$ with an interval on $\mathbf{R}$ by $C_{n}(\Omega ; I)$.

We shall say that a set $H \subset C_{n}(\Omega)$ has a covering $\left\{r_{j}, R_{j}\right\}$ if there exists a sequence of balls $\left\{B_{j}\right\}$ with centers in $C_{n}(\Omega)$ such that $H \subset \bigcup_{j=0}^{\infty} B_{j}$, where $r_{j}$ is the radius of $B_{j}$ and $R_{j}$ is the distance from the origin to the center of $B_{j}$.

From now on, we always assume $D=C_{n}(\Omega)$. For the sake of brevity, we shall write $G_{\Omega}^{a}(P, Q)$ instead of $G_{C_{n}(\Omega)}^{a}(P, Q)$. Throughout this paper, let $c$ denote various positive constants, because we do not need to specify them. Moreover, $\epsilon$ appearing in the expression in the following sections will be a sufficiently small positive number.

Let $\Omega$ be a domain on $\mathbf{S}^{n-1}$ with smooth boundary. Consider the Dirichlet problem

$$
\begin{aligned}
& \left(\Lambda_{n}+\lambda\right) \varphi=0 \quad \text { on } \Omega, \\
& \varphi=0 \quad \text { on } \partial \Omega,
\end{aligned}
$$

where $\Lambda_{n}$ is the spherical part of the Laplace operator $\Delta_{n}$

$$
\Delta_{n}=\frac{n-1}{r} \frac{\partial}{\partial r}+\frac{\partial^{2}}{\partial r^{2}}+\frac{\Lambda_{n}}{r^{2}}
$$

We denote the least positive eigenvalue of this boundary value problem by $\lambda$ and the normalized positive eigenfunction corresponding to $\lambda$ by $\varphi(\Theta)$. In order to ensure the existence of $\lambda$ and a smooth $\varphi(\Theta)$, we put a rather strong assumption on $\Omega$ : if $n \geq 3$, then $\Omega$ is a $C^{2, \alpha}$-domain $(0<\alpha<1)$ on $\mathbf{S}^{n-1}$ surrounded by a finite number of mutually disjoint closed hypersurfaces (e.g., see [3, pp.88-89] for the definition of $C^{2, \alpha}$-domain).

For any $(1, \Theta) \in \Omega$, we have (see $[4$, pp.7-8])

$$
c^{-1} r \varphi(\Theta) \leq \delta(P) \leq \operatorname{cr} \varphi(\Theta),
$$

where $P=(r, \Theta) \in C_{n}(\Omega)$ and $\delta(P)=\operatorname{dist}\left(P, \partial C_{n}(\Omega)\right)$.

Solutions of an ordinary differential equation

$$
-Q^{\prime \prime}(r)-\frac{n-1}{r} Q^{\prime}(r)+\left(\frac{\lambda}{r^{2}}+a(r)\right) Q(r)=0, \quad 0<r<\infty .
$$

It is known (see, for example, [5]) that if the potential $a \in \mathscr{A}_{a}$, then equation (2) has a fundamental system of positive solutions $\{V, W\}$ such that $V$ and $W$ are increasing and decreasing, respectively. 
We will also consider the class $\mathscr{B}_{a}$, consisting of the potentials $a \in \mathscr{A}_{a}$, such that there exists the finite limit $\lim _{r \rightarrow \infty} r^{2} a(r)=k \in[0, \infty)$ and, moreover, $r^{-1}\left|r^{2} a(r)-k\right| \in L(1, \infty)$. If $a \in \mathscr{B}_{a}$, then the (sub)superfunctions are continuous (see [6]).

In the rest of paper, we assume that $a \in \mathscr{B}_{a}$ and we shall suppress this assumption for simplicity.

Denote

$$
\iota_{k}^{ \pm}=\frac{2-n \pm \sqrt{(n-2)^{2}+4(k+\lambda)}}{2}
$$

then the solutions to equation (2) have the asymptotic (see [3])

$$
c^{-1} r^{r_{k}^{+}} \leq V(r) \leq c r^{l_{k}^{+}}, \quad c^{-1} r^{l_{k}^{-}} \leq W(r) \leq c r^{l_{k}^{-}}, \quad \text { as } r \rightarrow \infty .
$$

Let $v$ be any positive measure on $C_{n}(\Omega)$ such that the Green $a$-potential

$$
G_{\Omega}^{a} v(P)=\int_{C_{n}(\Omega)} G_{\Omega}^{a}(P, Q) d v(Q) \not \equiv+\infty
$$

for any $P \in C_{n}(\Omega)$. Then the positive measure $m(v)$ on $\mathbf{R}^{n}$ is defined by

$$
d m(v)(Q)= \begin{cases}W(t) \varphi(\Phi) d v(Q), & Q=(t, \Phi) \in C_{n}(\Omega ;(1,+\infty)) \\ 0, & Q \in \mathbf{R}^{n}-C_{n}(\Omega ;(1,+\infty)) .\end{cases}
$$

Remark 1 We remark that the total mass $m(v)$ is finite (see [2, Lemma 5]).

For each $P=(r, \Theta) \in \mathbf{R}^{n}-\{O\}$, the maximal function $M(P ; \lambda, \beta)$ is defined by

$$
M(P ; \lambda, \beta)=\sup _{0<\rho<\frac{r}{2}} \frac{\lambda(B(P, \rho))}{\rho^{\beta}},
$$

where $\beta \geq 0$ and $\lambda$ is a positive measure on $\mathbf{R}^{n}$. The set

$$
\left\{P=(r, \Theta) \in \mathbf{R}^{n}-\{O\} ; M(P ; \lambda, \beta) r^{\beta}>\epsilon\right\}
$$

is denoted by $E(\epsilon ; \lambda, \beta)$.

It is known that the Martin boundary of $C_{n}(\Omega)$ is the set $\partial C_{n}(\Omega) \cup\{\infty\}$, each of which is a minimal Martin boundary point. For $P \in C_{n}(\Omega)$ and $Q \in \partial C_{n}(\Omega) \cup\{\infty\}$, the Martin kernel can be defined by $M_{\Omega}^{a}(P, Q)$. If the reference point $P$ is chosen suitably, then we have

$$
M_{\Omega}^{a}(P, \infty)=V(r) \varphi(\Theta) \quad \text { and } \quad M_{\Omega}^{a}(P, O)=c W(r) \varphi(\Theta)
$$

for any $P=(r, \Theta) \in C_{n}(\Omega)$.

In [7], Long et al. introduced the notations of $a$-thin (with respect to the Schrödinger operator $S c h_{a}$ ) at a point, $a$-polar set (with respect to the Schrödinger operator $S c h_{a}$ ) and $a$-rarefied sets at infinity (with respect to the Schrödinger operator $S c h_{a}$ ), which generalized earlier notations obtained by Brelot and Miyamoto (see [8, 9]). A set $H$ in $\mathbf{R}^{n}$ is said 
to be $a$-thin at a point $Q$ if there is a fine neighborhood $E$ of $Q$ which does not intersect $H \backslash\{Q\}$. Otherwise $H$ is said to be not $a$-thin at $Q$ on $C_{n}(\Omega)$. A set $H$ in $\mathbf{R}^{n}$ is called a polar set if there is a superfunction $u$ on some open set $E$ such that $H \subset\{P \in E ; u(P)=\infty\}$. A subset $H$ of $C_{n}(\Omega)$ is said to be $a$-rarefied at infinity on $C_{n}(\Omega)$ if there exists a positive superfunction $v(P)$ on $C_{n}(\Omega)$ such that

$$
\inf _{P \in C_{n}(\Omega)} \frac{v(P)}{M_{\Omega}^{a}(P, \infty)} \equiv 0
$$

and

$$
H \subset\left\{P=(r, \Theta) \in C_{n}(\Omega) ; v(P) \geq V(r)\right\} .
$$

Let $H$ be a bounded subset of $C_{n}(\Omega)$. Then $\hat{R}_{M_{\Omega}^{a}(\cdot, \infty)}$ is bounded on $C_{n}(\Omega)$ and the greatest $a$-harmonic minorant of $\hat{R}_{M_{\Omega}^{a}(\cdot, \infty)}$ is zero. We see from the Riesz decomposition theorem (see [10, Theorem 2]) that there exists a unique positive measure $\lambda_{H}^{a}$ on $C_{n}(\Omega)$ such that (see $[7$, p.6])

$$
\hat{R}_{M_{\Omega}^{a}(\cdot, \infty)}^{H}(P)=G_{\Omega}^{a} \lambda_{H}^{a}(P)
$$

for any $P \in C_{n}(\Omega)$ and $\lambda_{H}^{a}$ is concentrated on $I_{H}$, where

$$
I_{H}=\left\{P \in C_{n}(\Omega) ; H \text { is not } a \text {-thin at } P\right\} .
$$

We denote the total mass $\lambda_{H}^{a}\left(C_{n}(\Omega)\right)$ of $\lambda_{H}^{a}$ by $\lambda_{\Omega}^{a}(H)$.

By using this positive measure $\lambda_{H}^{a}$ (with respect to the Schrödinger operator $S c h_{a}$ ), we can further define another measure $\eta_{H}^{a}$ on $C_{n}(\Omega)$ by

$$
d \eta_{H}^{a}(P)=M_{\Omega}^{a}(P, \infty) d \lambda_{H}^{a}(P)
$$

for any $P \in C_{n}(\Omega)$. It is easy to see that $\eta_{H}^{a}\left(C_{n}(\Omega)\right)<+\infty$.

Recently, Long et al. (see [7, Theorem 2.5]) gave a criterion for a subset $H$ of $C_{n}(\Omega)$ to be $a$-rarefied set at infinity.

Theorem A $A$ subset $H$ of $C_{n}(\Omega)$ is a-rarefied at infinity on $C_{n}(\Omega)$ if and only if

$$
\sum_{j=0}^{\infty} \lambda_{\Omega}^{a}\left(H_{j}\right) W\left(2^{j}\right)<\infty
$$

where $H_{j}=H \cap C_{n}\left(\Omega ;\left[2^{j}, 2^{j+1}\right)\right)$ and $j=0,1,2, \ldots$

In this paper, we shall obtain a series of new criteria for $a$-rarefied sets at infinity on $C_{n}(\Omega)$, which complement Theorem A. Our results are essentially based on Qiao and Deng, Ren and Zhao, Xue (see [2, 11-14]). In order to avoid complexity of our proofs, we shall assume $n \geq 3$. But our results in this paper are also true for $n=2$.

First we shall state Theorem 1, which is the main result in this paper. 
Theorem 1 A subset $H$ of $C_{n}(\Omega)$ is a-rarefied at infinity on $C_{n}(\Omega)$ if and only if there exists a positive measure $\xi_{H}^{a}$ on $C_{n}(\Omega)$ such that

$$
G_{\Omega}^{a} \xi_{H}^{a}(P) \not \equiv+\infty
$$

for any $P \in C_{n}(\Omega)$ and

$$
H \subset\left\{P=(r, \Theta) \in C_{n}(\Omega) ; G_{\Omega}^{a} \xi_{H}^{a}(P) \geq V(r)\right\}
$$

Next we give the geometrical property of $a$-rarefied sets at infinity.

Theorem 2 If a subset $H$ of $C_{n}(\Omega)$ is a-rarefied at infinity on $C_{n}(\Omega)$, then $H$ has a covering $\left\{r_{j}, R_{j}\right\}(j=0,1,2, \ldots)$ satisfying

$$
\sum_{j=0}^{\infty}\left(\frac{r_{j}}{R_{j}}\right) V\left(\frac{R_{j}}{r_{j}}\right) W\left(\frac{R_{j}}{r_{j}}\right)<\infty .
$$

Finally, by an example we show that the reverse of Theorem 2 is not true.

Example Put

$$
r_{j}=3 \cdot 2^{j-1} \cdot j^{\frac{1}{2-n}} \quad \text { and } \quad R_{j}=3 \cdot 2^{j-1} \quad(j=1,2,3, \ldots) .
$$

A covering $\left\{r_{j}, R_{j}\right\}$ satisfies

$$
\sum_{j=1}^{\infty}\left(\frac{r_{j}}{R_{j}}\right) V\left(\frac{R_{j}}{r_{j}}\right) W\left(\frac{R_{j}}{r_{j}}\right) \leq c \sum_{j=1}^{\infty}\left(\frac{r_{j}}{R_{j}}\right)^{n-1}=c \sum_{j=1}^{\infty} j^{\frac{n-1}{2-n}<+\infty}
$$

from equation (3).

Let $C_{n}\left(\Omega^{\prime}\right)$ be a subset of $C_{n}(\Omega)$, i.e., $\bar{\Omega}^{\prime} \subset \Omega$. Suppose that this covering is located as follows: there is an integer $j_{0}$ such that $B_{j} \subset C_{n}\left(\Omega^{\prime}\right)$ and $R_{j}>2 r_{j}$ for $j \geq j_{0}$. Then the set $H=\bigcup_{j=j_{0}}^{\infty} B_{j}$ is not $a$-rarefied at infinity on $C_{n}(\Omega)$. This fact will be proved in Section 5 .

\section{Lemmas}

Lemma 1 (see [1, Ch. 11] and [15, Lemma 4])

$$
\begin{aligned}
& G_{\Omega}^{a}(P, Q) \leq c V(t) W(r) \varphi(\Theta) \varphi(\Phi) \\
& \left(\text { resp. } G_{\Omega}^{a}(P, Q) \leq c V(r) W(t) \varphi(\Theta) \varphi(\Phi)\right)
\end{aligned}
$$

for any $P=(r, \Theta) \in C_{n}(\Omega)$ and any $Q=(t, \Phi) \in C_{n}(\Omega)$ satisfying $r \geq 2 t($ resp. $t \geq 2 r)$.

Lemma 2 (see [2, Lemma 5]) Let $v$ be a positive measure on $C_{n}(\Omega)$ such that there is a sequence of points $P_{i}=\left(r_{i}, \Theta_{i}\right) \in C_{n}(\Omega), r_{i} \rightarrow+\infty(i \rightarrow+\infty)$ satisfying $G_{\Omega}^{a} v\left(P_{i}\right)<+\infty$ $\left(i=1,2, \ldots ; Q \in C_{n}(\Omega)\right)$. Then, for a positive number $L$,

$$
\int_{C_{n}(\Omega ;(L,+\infty))} W(t) \varphi(\Phi) d v(Q)<+\infty
$$


and

$$
\lim _{R \rightarrow+\infty} \frac{W(R)}{V(R)} \int_{C_{n}(\Omega ;(0, R))} V(t) \varphi(\Phi) d \nu(Q)=0 .
$$

Lemma 3 (see [2, Theorem 3]) Let $v$ be any positive measure on $C_{n}(\Omega)$ such that $G_{\Omega}^{a} v(P) \not \equiv$ $+\infty$ for any $P \in C_{n}(\Omega)$. Then, for a sufficiently large $L$,

$$
\left\{P=(r, \Theta) \in C_{n}(\Omega ;(L,+\infty)) ; G_{\Omega}^{a} \nu(P) \geq V(r) \varphi(\Theta)\right\} \subset E(\epsilon ; m(\nu), n-1) .
$$

Lemma 4 (see [2, Lemma 6]) Let $\lambda$ be any positive measure on $\mathbf{R}^{n}$ having finite total mass. Then $E(\epsilon ; \lambda, n-1)$ has a covering $\left\{r_{j}, R_{j}\right\}(j=1,2, \ldots)$ satisfying

$$
\sum_{j=1}^{\infty}\left(\frac{r_{j}}{R_{j}}\right) V\left(\frac{R_{j}}{r_{j}}\right) W\left(\frac{R_{j}}{r_{j}}\right)<\infty
$$

\section{Proof of Theorem 1}

Suppose that

$$
H \subset \Pi\left(\xi_{H}^{a}\right)=\left\{P=(r, \Theta) \in C_{n}(\Omega) ; G_{\Omega}^{a} \xi_{H}^{a}(P) \geq V(r)\right\}
$$

for a positive measure $\xi_{H}^{a}$ on $C_{n}(\Omega)$ satisfying equation (6).

We write

$$
G_{\Omega}^{a} v(P)=G_{\Omega}^{a}(1, j)(P)+G_{\Omega}^{a}(2, j)(P)+G_{\Omega}^{a}(3, j)(P),
$$

where

$$
\begin{aligned}
& G_{\Omega}^{a}(1, j)(P)=\int_{C_{n}\left(\Omega ;\left(0,2^{j-1}\right)\right)} G_{\Omega}^{a}(P, Q) d \nu(Q), \\
& G_{\Omega}^{a}(2, j)(P)=\int_{C_{n}\left(\Omega ;\left[j^{-1}, 2^{j+2}\right)\right)} G_{\Omega}^{a}(P, Q) d \nu(Q)
\end{aligned}
$$

and

$$
G_{\Omega}^{a}(3, j)(P)=\int_{C_{n}\left(\Omega ;\left[2^{j+2}, \infty\right)\right)} G_{\Omega}^{a}(P, Q) d \nu(Q) .
$$

Now we shall show the existence of an integer $N$ such that for any integer $j(\geq N)$, we have

$$
\Pi\left(\xi_{H}^{a}\right)(j) \subset\left\{P=(r, \Theta) \in C_{n}\left(\Omega ;\left[2^{j}, 2^{j+1}\right)\right) ; 2 G_{\Omega}^{a}(2, j)(P) \geq V(r)\right\}
$$

for any integer $j(\geq N)$.

For any $P=(r, \Theta) \in C_{n}\left(\Omega ;\left[2^{j}, 2^{j+1}\right)\right)$, we have

$$
G_{\Omega}^{a}(1, j)(P) \leq c W(r) \varphi(\Theta) \int_{C_{n}\left(\Omega ;\left(0,2^{j-1}\right)\right)} V(t) \varphi(\Phi) d \nu(Q)
$$


and

$$
G_{\Omega}^{a}(3, j)(P) \leq c V(r) \varphi(\Theta) \int_{C_{n}\left(\Omega ;\left[2^{j+2}, \infty\right)\right)} d m(v)(Q)
$$

from Lemma 1.

By applying Lemma 2 , we can take an integer $N$ such that for any $j(\geq N)$,

$$
W\left(2^{j}\right) V^{-1}\left(2^{j}\right) \int_{C_{n}\left(\Omega ;\left(0,2^{j-1}\right)\right)} V(t) \varphi(\Phi) d v(Q) \leq \frac{1}{4 c}
$$

and

$$
\int_{C_{n}\left(\Omega ;\left[2^{j+2}, \infty\right)\right)} d m(v)(Q) \leq \frac{1}{4 c}
$$

Thus we obtain

$$
4 G_{\Omega}^{a}(1, j)(P) \leq V(r) \varphi(\Theta)
$$

and

$$
4 G_{\Omega}^{a}(3, j)(P) \leq V(r) \varphi(\Theta)
$$

for any $P=(r, \Theta) \in C_{n}\left(\Omega ;\left[2^{j}, 2^{j+1}\right)\right)$, where $j \geq N$.

Thus, if $P=(r, \Theta) \in \Pi(v)(j)(j \geq N)$, then we obtain

$$
2 G_{\Omega}^{a}(1, j)(P) \geq V(r) \varphi(\Theta)
$$

from equations (12) and (13), which gives equation (11).

From equations (4), (7) and (11), we have

$$
G_{\Omega}^{a}(2, j)(P)=\int_{C_{n}(\Omega)} G_{\Omega}^{a}(P, Q) d \tau_{j}^{a}(Q) \geq M_{\Omega}^{a}(P, \infty),
$$

where $P \in I_{j}(j \geq N)$ and

$$
d \tau_{j}^{a}(Q)= \begin{cases}2^{1-j} d \xi_{H}^{a}(Q), & Q \in C_{n}\left(\Omega ;\left[2^{j-1}, 2^{j+2}\right)\right), \\ 0, & Q \in C_{n}\left(\Omega ;\left(0,2^{j-1}\right)\right) \cup C_{n}\left(\Omega ;\left[2^{j+2}, \infty\right)\right) .\end{cases}
$$

And then we obtain

$$
\eta_{H_{j}}^{a}\left(C_{n}(\Omega)\right) \leq \int_{C_{n}(\Omega)} V(t) \varphi(\Phi) d \tau_{j}^{a}(Q)=\int_{C_{n}\left(\Omega ;\left[2^{j-1}, 2^{j+2}\right)\right)} V(t) \varphi(\Phi) d \xi_{H}^{a}(Q)
$$

for $j \geq N$. Then we have

$$
\sum_{j=N}^{\infty} \lambda_{\Omega}^{a}\left(H_{j}\right) W\left(2^{j}\right)=\sum_{j=N}^{\infty} \eta_{H_{j}}^{a}\left(C_{n}(\Omega)\right) W\left(2^{j}\right) \leq c \int_{C_{n}\left(\Omega ;\left[2^{N-1}, \infty\right)\right)} d m\left(\xi_{H}^{a}\right)
$$


in which the last integral is finite by Remark 1 . And hence $H$ is $a$-rarefied set at infinity from Theorem A.

Suppose that

$$
\sum_{j=0}^{\infty} \lambda_{\Omega}^{a}\left(H_{j}\right) W\left(2^{j}\right)<\infty
$$

Consider a function $f_{H}^{a}(P)$ on $C_{n}(\Omega)$ defined by

$$
f_{H}^{a}(P)=\sum_{j=-1}^{\infty} \hat{R}_{M_{\Omega}^{a}(\cdot, \infty)}^{H_{j}}(P)
$$

for any $P \in C_{n}(\Omega)$, where $H_{-1}=H \cap C_{n}(\Omega ;(0,1))$.

If we put $\mu_{H}^{a}(1)(P)=\sum_{j=-1}^{\infty} \lambda_{H_{j}}^{a}(P)$, then from equation (5) we have that

$$
f_{H}^{a}(P)=\int_{C_{n}(\Omega)} G_{\Omega}^{a}(P, Q) d \mu_{H}^{a}(1)(Q)
$$

for any $P \in C_{n}(\Omega)$.

Next we shall show that $f_{H}^{a}(P)$ is always finite on $C_{n}(\Omega)$. Take any point $P=(r, \Theta) \in C_{n}(\Omega)$ and a positive integer $j(P)$ satisfying $r \leq 2^{j(P)+1}$. We write

$$
f_{H}^{a}(P)=f_{H}^{a}(1)(P)+f_{H}^{a}(2)(P),
$$

where

$$
\begin{aligned}
& f_{H}^{a}(1)(P)=\sum_{j=-1}^{j(P)+1} \int_{C_{n}(\Omega)} G_{\Omega}^{a}(P, Q) d \lambda_{H_{j}}^{a}(Q) \quad \text { and } \\
& f_{H}^{a}(2)(P)=\sum_{j=j(P)+2}^{\infty} \int_{C_{n}(\Omega)} G_{\Omega}^{a}(P, Q) d \lambda_{H_{j}}^{a}(Q) .
\end{aligned}
$$

Since $\lambda_{H_{j}}^{a}$ is concentrated on $I_{H_{j}} \subset \bar{H}_{j} \cap C_{n}(\Omega)$, we have that

$$
\begin{aligned}
\int_{C_{n}(\Omega)} G_{\Omega}^{a}(P, Q) d \lambda_{H_{j}}^{a}(Q) & \leq c V(r) \varphi(\Theta) \int_{C_{n}(\Omega)} W(t) \varphi(\Phi) d \lambda_{H_{j}}^{a}(t, \Phi) \\
& \leq c V(r) \varphi(\Theta) W\left(2^{j}\right) V^{-1}\left(2^{j}\right) \int_{C_{n}(\Omega)} V(t) \varphi(\Phi) d \lambda_{H_{j}}^{a}(t, \Phi)
\end{aligned}
$$

for $j \geq j(P)+2$. Hence we have

$$
f_{H}^{a}(2)(P) \leq c V(r) \varphi(\Theta) \sum_{j=j(P)+2}^{\infty} \eta_{H_{j}}^{a}\left(C_{n}(\Omega)\right) W\left(2^{j}\right) V^{-1}\left(2^{j}\right),
$$

which, together with Theorem A, shows that $f_{H}^{a}(2)(P)$ is finite and hence $f_{H}^{a}(P)$ is also finite for any $P \in C_{n}(\Omega)$.

Since

$$
\hat{R}_{M_{\Omega}^{a}(\cdot, \infty)}^{H_{j}}(P)=M_{\Omega}^{a}(P, \infty)
$$


holds on $I_{H_{j}}$ and $I_{H_{j}} \subset \bar{H}_{j} \cap C_{n}(\Omega)$, we see that for any $P=(r, \Theta) \in I_{H_{j}}(j=-1,0,1,2,3, \ldots)$

$$
f_{H}^{a}(P) \geq c \hat{R}_{M_{\Omega}^{a}(\cdot, \infty)}^{H_{j}}(P) \geq V(r) \varphi(\Theta) .
$$

And hence equation (15) also holds for any $P=(r, \Theta) \in H^{\prime}=\bigcup_{j=-1}^{\infty} I_{H_{j}}$. Since $H^{\prime}$ is equal to $H$ except a polar set $H^{0}$, we can take another positive superfunction $f_{H}^{a}(3)(P)$ on $C_{n}(\Omega)$ such that $f_{H}^{a}(3)(P)=G_{\Omega}^{a} \mu_{H}^{a}(2)(P)$ with a positive measure $\mu_{H}^{a}(2)(P)$ on $C_{n}(\Omega)$ and $f_{H}^{a}(3)(P)$ is identically $+\infty$ on $H^{0}$.

Finally, we can define a positive superfunction $g$ on $C_{n}(\Omega)$ by $g(P)=f_{H}^{a}(P)+f_{H}^{a}(3)(P)=$ $G_{\Omega}^{a} \xi_{H}^{a}(P)$ for any $P \in C_{n}(\Omega)$ with $\xi_{H}^{a}=\mu_{H}^{a}(1)+\mu_{H}^{a}(2)$. Also we see from equation (15) that equations (6) and (7) hold.

Thus we complete the proof of Theorem 1.

\section{Proof of Theorem 2}

From Theorem 1 and Lemma 3, we have a positive number $L$ such that

$$
H \cap C_{n}(\Omega ;(L,+\infty)) \subset E\left(\epsilon ; m\left(\xi_{H}^{a}\right), n-1\right) .
$$

Hence by Remark 1 and Lemma $4, E\left(\epsilon ; m\left(\xi_{H}^{a}\right), n-1\right)$ has a covering $\left\{r_{j}, R_{j}\right\}(j=1,2,3, \ldots)$ satisfying equation (9) and hence $H$ has also a covering $\left\{r_{j}, R_{j}\right\}(j=0,1,2,3, \ldots)$ with an additional finite $B_{0}$ covering $C_{n}(\Omega ;(0, L])$, satisfying equation (8), which is the conclusion of Theorem 2.

\section{Proof of an example}

Since $\varphi(\Theta) \geq c$ for any $\Theta \in \Omega^{\prime}$, we have $M_{\Omega}^{a}(P, \infty) \geq c V\left(R_{j}\right)$ for any $P \in \bar{B}_{j}$, where $j \geq j_{0}$. Hence we have

$$
\hat{R}_{M_{\Omega}^{a}(\cdot, \infty)}^{B_{j}}(P) \geq c V\left(R_{j}\right)
$$

for any $P \in \bar{B}_{j}$, where $j \geq j_{0}$.

Take a measure $\delta$ on $C_{n}(\Omega)$, supp $\delta \subset \bar{B}_{j}, \delta\left(\bar{B}_{j}\right)=1$ such that

$$
\int_{C_{n}(\Omega)}|P-Q|^{2-n} d \delta(P)=\left\{\operatorname{Cap}\left(\bar{B}_{j}\right)\right\}^{-1}
$$

for any $Q \in \bar{B}_{j}$, where Cap denotes the Newton capacity. Since

$$
G_{\Omega}^{a}(P, Q) \leq|P-Q|^{2-n}
$$

for any $P \in C_{n}(\Omega)$ and $Q \in C_{n}(\Omega)$ (see [16], the case $n=2$ is implicitly contained in [17]),

$$
\begin{aligned}
\left\{\operatorname{Cap}\left(\bar{B}_{j}\right)\right\}^{-1} \lambda_{B_{j}}^{a}\left(C_{n}(\Omega)\right) & =\int\left(\int|P-Q|^{2-n} d \delta(P)\right) d \lambda_{B_{j}}^{a}(Q) \\
& \geq \int\left(\int G_{\Omega}^{a}(P, Q) d \lambda_{B_{j}}^{a}(Q)\right) d \delta(P) \\
& =\int \hat{R}_{M_{\Omega}^{a}(\cdot, \infty)}^{B_{j}} d \delta(P) \\
& \geq c V\left(R_{j}\right) \delta\left(\bar{B}_{j}\right)=c V\left(R_{j}\right)
\end{aligned}
$$


from equations (16) and (17). Hence we have

$$
\lambda_{B_{j}}^{a}\left(C_{n}(\Omega)\right) \geq c \operatorname{Cap}\left(\bar{B}_{j}\right) V\left(R_{j}\right) \geq c r_{j}^{n-2} V\left(R_{j}\right) .
$$

If we observe $\lambda_{H_{j}}^{a}\left(C_{n}(\Omega)\right)=\lambda_{B_{j}}^{a}\left(C_{n}(\Omega)\right)$, then we have by equation (3)

$$
\sum_{j=j_{0}}^{\infty} W\left(2^{j}\right) \lambda_{H_{j}}^{a}\left(C_{n}(\Omega)\right) \geq c \sum_{j=j_{0}}^{\infty}\left(\frac{r_{j}}{R_{j}}\right)^{n-2}=c \sum_{j=j_{0}}^{\infty} \frac{1}{j}=+\infty,
$$

from which it follows by Theorem A that $H$ is not $a$-rarefied at infinity on $C_{n}(\Omega)$.

\section{Competing interests}

The author declares that they have no competing interests.

\section{Acknowledgements}

This work was supported by the National Natural Science Foundation of China under Grants Nos. 11301140 and U1304102.

\section{Received: 26 March 2014 Accepted: 30 May 2014 Published: 18 July 2014}

\section{References}

1. Escassut, A, Tutshke, W, Yang, CC: Some Topics on Value Distribution and Differentiability in Complex and p-Adic Analysis. Science Press, Beijing (2008)

2. Qiao, L, Deng, GT: Integral representations and growth properties for a class of superfunctions in a cone. Taiwan. J. Math. 15, 2213-2233 (2011)

3. Gilbarg, D, Trudinger, NS: Elliptic Partial Differential Equations of Second Order. Springer, Berlin (1977)

4. Courant, R, Hilbert, D: Methods of Mathematical Physics, vol. 1. Interscience, New York (2008)

5. Verzhbinskii, GM, Maz'ya, VG: Asymptotic behavior of solutions of elliptic equations of the second order close to a boundary. I. Sib. Mat. Zh. 12, 874-899 (1971)

6. Simon, B: Schrödinger semigroups. Bull. Am. Math. Soc. 7, 447-526 (1982)

7. Long, $\mathrm{PH}, \mathrm{Gao}, \mathrm{ZQ}$, Deng, GT: Criteria of Wiener type for minimally thin sets and rarefied sets associated with the stationary Schrödinger operator in a cone. Abstr. Appl. Anal. 2012, Article ID 453891 (2012)

8. Brelot, M: On Topologies and Boundaries in Potential Theory. Lecture Notes in Mathematics, vol. 175. Springer, Berlin (1971)

9. Miyamoto, I, Yoshida, H: Two criterions of Wiener type for minimally thin sets and rarefied sets in a cone. J. Math. Soc. Jpn. 54, 487-512 (2002)

10. Qiao, L, Pan, GS: Generalization of the Phragmén-Lindelöf theorems for subfunctions. Int. J. Math. 24(8), 1350062 (2013). doi:10.1142/S0129167X13500626

11. Qiao, L, Pan, GS: Integral representations of generalized harmonic functions. Taiwan. J. Math. 17(5), 1503-1521 (2013)

12. Ren, YD: Solving integral representations problems for the stationary Schrödinger equation. Abstr. Appl. Anal. 2013, Article ID 715252 (2013)

13. Zhao, T: Minimally thin sets associated with the stationary Schrödinger operator. J. Inequal. Appl. 2014,67 (2014)

14. Xue, GX: A remark on the a-minimally thin sets associated with the Schrödinger operator. Bound. Value Probl. 2014, $133(2014)$

15. Azarin, VS: Generalization of a theorem of Hayman on subharmonic functions in an $m$-dimensional cone. Transl. Am. Math. Soc. 80, 119-138 (1969)

16. Cranston, M: Conditional Brownian motion, Whitney squares and the conditional gauge theorem. In: Seminar on Stochastic Processes, 1988, pp. 109-119. Birkhäuser, Basel (1989)

17. Cranston, M, Fabes, E, Zhao, Z: Conditional gauge and potential theory for the Schrödinger operator. Trans. Am. Math. Soc. 307, 415-425 (1964)

doi:10.1186/1029-242X-2014-247

Cite this article as: Xue: Rarefied sets at infinity associated with the Schrödinger operator. Journal of Inequalities and Applications 2014 2014:247. 